\title{
Evaluation of marine sediments as microbial sources for methane production from brown algae under high salinity
}

Toyokazu Miura ${ }^{1,3}$, Akihisa Kita ${ }^{1,3}$, Yoshiko Okamura ${ }^{1,3}$, Tsunehiro Aki ${ }^{1,3}$, Yukihiko Matsumura $^{2,3}$, Takahisa Tajima ${ }^{1,3}$, Junichi Kato ${ }^{1}$, Yutaka Nakashimada ${ }^{1,3 *}$

${ }^{1}$ Department of Molecular Biotechnology, Graduate School of Advanced Sciences of Matter, Hiroshima University, 1-3-1 Kagamiyama, Higashi-Hiroshima 739-8530, Japan

${ }^{2}$ Division of Energy and Environmental Engineering, Institute of Engineering, Hiroshima University, 1-4-1 Kagamiyama, Higashi-Hiroshima 739-8527, Japan

${ }^{3}$ CREST, JST

* Corresponding author. Tel./fax: +81 824244443.

E-mail address: nyutaka@hiroshima-u.ac.jp 


\begin{abstract}
Various marine sediments were evaluated as promising microbial sources for methane fermentation of Saccharina japonica, a brown alga, at seawater salinity. All marine sediments tested produced mainly acetate among volatile fatty acids. One marine sediment completely converted the produced volatile fatty acids to methane in a short period. Archaeal community analysis revealed that acetoclastic methanogens belonging to the Methanosarcina genus dominated after cultivation. Measurement of the specific conversion rate at each step of methane production under saline conditions demonstrated that the marine sediments had higher conversion rates of butyrate and acetate than mesophilic methanogenic granules. These results clearly show that marine sediments can be used as microbial sources for methane production from algae under high-salt conditions without dilution.
\end{abstract}

Keywords: Brown algae, Marine macroalgae, Marine sediments, Methane production, Saccharina japonica

\title{
1. Introduction
}

Methane can be produced from biomass by anaerobic microbial conversion. Many organic components included in biomass, e.g., sugar, protein, and lipid, can be utilized for methane production. Among biomass types, marine macroalgae are an attractive 
feedstock for methane fermentation because they do not compete with agricultural land, and they have a lower lignin content than terrestrial lignocellulose, resulting in facile degradation (Wei et al., 2013). In marine macroalgae, however, the high salt content in the biomass can inhibit microbial methane production (Demirbas, 2010). Wet marine macroalgae contain approximately 3\% ash (Roesijadi et al., 2010); thus, their salt concentration can reach 3\%, which is similar to the salinity of seawater, during methane production. Dilution of marine macroalgae with water can solve this problem; however, such dilution leads to an increase in the cost of methane production because of the increased use of water (Shi et al., 2014). Thus, it would be ideal to use marine macroalgae as the substrate for methane production without dilution.

To produce methane from marine macroalgae without dilution, salt-tolerant microorganisms are needed. One possible approach is to use marine sediments as the source of the microorganisms. It has been reported that methane was produced from green algae in seawater by using a marine sediment and a sludge of nonmarine origin, and the methane yield obtained by using the marine sediment was higher than that obtained by using an inoculum of nonmarine origin (Schramm \& Lehnberg, 1984). Thus, it was considered that marine sediments could be effectively used as microbial sources for methane production from macroalgae at seawater salinity.

Methane is produced from organic matter in three steps, namely, hydrolysis/acidogenesis, acetogenesis, and methanogenesis. In the first step, organic matter is converted to volatile fatty acids (VFAs) by various bacteria. In the second step, VFAs other than acetate, namely propionate and butyrate, are converted to acetate and hydrogen by propionate- and butyrate-oxidizing bacteria, respectively. In the final step, hydrogen and carbon dioxide are converted to methane by hydrogenotrophic 
methanogens, and acetate is converted to methane and carbon dioxide by acetoclastic methanogens. Differences in the activity in the three steps affect the production of methane. Thus, it is important to understand the activity involved in methane production. However, for methane production from macroalgae by marine sediments at seawater salinity, there have been no reports on the conversion rate at each step of methane production.

In this study, therefore, various marine sediments were used as microbial sources for methane production from marine macroalgae at seawater salinity to measure the activity in three steps of methane production. Saccharina japonica, a brown alga and the most-produced macroalgae in the world (Jung et al., 2013), was used as the substrate for methane production. Methane production was conducted in a medium containing $30 \mathrm{~g}$ $\mathrm{NaCl} / \mathrm{L}$, which was similar to seawater salinity (Feijoo et al., 1995). Furthermore, the conversion rates of the second and third steps in methane production from the brown alga were measured to identify the rate-limiting step.

\section{Materials and Methods}

2.1. Materials

Dried S. japonica was purchased from a commission agent handling seafood. The total solid (TS), volatile solid (VS), and ash content of the brown alga were 94.2, 73.3, and 21.0 wt.\%, respectively. The chemical oxygen demand (COD) of the dried seaweed was $1,030 \mathrm{mg} / \mathrm{g}-\mathrm{TS}$. Alga milled to a size less than $0.7 \mathrm{~mm}$ was used in the experiments. 
Marine sediments and mesophilic methanogenic granules were used as microbial sources as listed in Table 1.

\subsection{Cultivation}

Microbial sources were added to a container, and dilution medium in an anaerobic state was added. The anaerobic state was induced by heating in a boiling water bath for $30 \mathrm{~min}$ and cooling on ice with continuous bubbling of $\mathrm{N}_{2} / \mathrm{CO}_{2}(80: 20)$ for $30 \mathrm{~min}$. The medium was dispensed to the container containing the sediments with continuous bubbling of $\mathrm{CO}_{2}$. The container was sealed with butyl rubber and then incubated at 37 ${ }^{\circ} \mathrm{C}$.

The dilution medium used for the cultivation of microbial sources with the brown alga had the following composition: $\left(\mathrm{NH}_{4}\right)_{2} \mathrm{SO}_{4}, 5 \mathrm{~g} / \mathrm{L} ; \mathrm{Na}_{2} \mathrm{MoO}_{4} \cdot 2 \mathrm{H}_{2} \mathrm{O}, 0.12 \mathrm{~g} / \mathrm{L}$; $\mathrm{Fe}\left(\mathrm{NH}_{4}\right)_{2} \mathrm{SO}_{4} \bullet 6 \mathrm{H}_{2} \mathrm{O}, 0.039 \mathrm{~g} / \mathrm{L} ; \mathrm{Co}\left(\mathrm{NO}_{3}\right)_{2} \bullet 6 \mathrm{H}_{2} \mathrm{O}, 0.029 \mathrm{~g} / \mathrm{L} ; \mathrm{CaCl}_{2} \cdot 2 \mathrm{H}_{2} \mathrm{O}, 0.021 \mathrm{~g} / \mathrm{L}$; $\mathrm{MgSO}_{4} \cdot 7 \mathrm{H}_{2} \mathrm{O}, 0.25 \mathrm{~g} / \mathrm{L} ; \mathrm{NaHCO}_{3}, 10 \mathrm{~g} / \mathrm{L} ; \mathrm{NaCl}, 30 \mathrm{~g} / \mathrm{L}$; vitamin solution, $10 \mathrm{~mL} / \mathrm{L}$; and trace element solution, $10 \mathrm{~mL} / \mathrm{L}$. Marine sediments that contained $1 \mathrm{~g}$ of TS or mesophilic granules that contained $0.03 \mathrm{~g}$ of TS were added to a test tube with $10 \mathrm{~mL}$ of the dilution medium that contained the alga at a concentration of $5 \mathrm{~g} / \mathrm{L}$. The tube was incubated with shaking. For subculture, $5 \mathrm{~mL}$ of the culture was inoculated to $50 \mathrm{~mL}$ of medium in a $125-\mathrm{mL}$ vial.

The dilution medium used for cultivation with VFA or hydrogen had the following composition: $\mathrm{NH}_{4} \mathrm{Cl}, 1 \mathrm{~g} / \mathrm{L} ; \mathrm{KH}_{2} \mathrm{PO}_{4}, 0.3 \mathrm{~g} / \mathrm{L} ; \mathrm{CaCl}_{2} \cdot 2 \mathrm{H}_{2} \mathrm{O}, 0.08 \mathrm{~g} / \mathrm{L} ; \mathrm{MgCl}_{2} \bullet 6 \mathrm{H}_{2} \mathrm{O}, 0.1$ g/L; $\mathrm{KHCO}_{3}, 4$ g/L; $\mathrm{NaCl}, 30 \mathrm{~g} / \mathrm{L}$; vitamin solution, $10 \mathrm{~mL} / \mathrm{L}$; and trace element solution, $10 \mathrm{~mL} / \mathrm{L}$. 
Acetogenesis was conducted with the dilution medium that contained propionate or butyrate at a concentration of $5 \mathrm{~g} / \mathrm{L} ; 10 \mathrm{~mL}$ of hydrogen-consuming culture from marine sediments and $5 \mathrm{~mL}$ of hydrogen-consuming culture from the mesophilic granules were added to a $125-\mathrm{mL}$ vial with 40 and $20 \mathrm{~mL}$ of the medium, respectively.

Hydrogen-consuming culture was obtained as described below to prevent inhibition of hydrogen-forming oxidation of VFAs by residual hydrogen partial pressure. The vial was incubated under static conditions.

For hydrogenotrophic methanogenesis, marine sediments that contained $2.5 \mathrm{~g}$ of TS and the mesophilic granules that contained $0.63 \mathrm{~g}$ of TS were added to a $125-\mathrm{mL}$ vial with 25 and $12.5 \mathrm{~mL}$ of the dilution medium, respectively. The headspace of the vial was flushed with $\mathrm{N}_{2} / \mathrm{CO}_{2}(80: 20)$, and then pressurized with $\mathrm{H}_{2} / \mathrm{CO}_{2}(80: 20)$ to 1.5 or 2 atm. The vial was incubated with shaking.

Acetoclastic methanogenesis was conducted with dilution medium that contained acetate at a concentration of $5 \mathrm{~g} / \mathrm{L}$. Marine sediments that contained 10 and $60 \mathrm{~g}$ of TS were added to a $125-\mathrm{mL}$ vial with $50 \mathrm{~mL}$ of the medium and a $750-\mathrm{mL}$ vial with $300 \mathrm{~mL}$ of the medium, respectively. Mesophilic granules that contained $0.25 \mathrm{~g}$ of TS were added to a $125-\mathrm{mL}$ vial with $25 \mathrm{~mL}$ of the medium. The vial was incubated under static conditions.

The specific conversion rate was estimated from the maximum slope calculated using an approximation formula. The formula was obtained by fitting a third-order polynomial trendline to the experimental data points using Microsoft Excel.

The VFAs produced in the subculture were converted to COD, and the amount of VFA-COD per unit amount of VS of the inoculum was plotted against the incubation period to calculate the VFA formation rate. In other conversions, the substrates 
consumed were converted to substrate-COD per unit amount of VS of inoculum to calculate the substrate consumption rate.

\subsection{Analytical methods}

TS content was measured by drying wet marine sediment at $105^{\circ} \mathrm{C}$ for $>4 \mathrm{~h}$. VS content was calculated as the ash-free dry weight. Ash content was measured by heating dried marine sediment at $600{ }^{\circ} \mathrm{C}$ for $3 \mathrm{~h}$. COD was analyzed by a commercially available kit according to the manufacturer's protocol (Hach, Loveland, CO, USA).

VFAs and other intermediate organic substances were quantified by high-performance liquid chromatography (LC-2000 Plus HPLC; Jasco, Tokyo, Japan) equipped with a refractive index detector (RI-2031 Plus; Jasco), Shodex RSpak KC-811 column (Showa Denko, Kanagawa, Japan), and a guard column (Shodex RSpak KC-G; Showa Denko) at $60{ }^{\circ} \mathrm{C}$. Ultrapure water containing $0.1 \%(\mathrm{v} / \mathrm{v})$ phosphoric acid was used as the mobile phase at a flow rate of $0.7 \mathrm{~mL} / \mathrm{min}$. Crotonate was used as an internal standard.

The gas composition in the headspace of the container was analyzed by gas chromatography (GC-8A; Shimadzu, Kyoto, Japan) equipped with a thermal conductivity detector and a stainless steel column packed with activated carbon, at $70{ }^{\circ} \mathrm{C}$. Argon was used as the carrier gas. The gas volume was measured by ejecting the gas in the headspace to a cylinder containing saturated aqueous $\mathrm{NaCl}$ (Abouelenien et al., 2010). The amount of the solution displaced with gas was measured as the gas volume. The gas volume measurement was initiated after the hydrogen produced was consumed. 
Genomic DNA was extracted from triplicate cultures or subcultures (500 $\mu \mathrm{L}$ each) by using the FastDNA SPIN Kit for Soil (Qbiogene, Carlsbad, CA, USA). The concentration of the extracted genomic DNA was quantified using a spectrophotometer. The DNA extracted from each culture or subculture was diluted to $10 \mathrm{ng} / \mathrm{L}$, and the diluted samples were combined. The DNA solution was used as the template for PCR, which was conducted in $200 \mu \mathrm{L}$ of a reaction mixture containing PCR buffer for KOD FX Neo, $0.4 \mathrm{mM}$ dNTPs, $0.3 \mu \mathrm{M}$ primers, $10 \mathrm{ng}$ of template, and $0.02 \mathrm{U} / \mu \mathrm{L}$ KOD FX Neo DNA polymerase (Toyobo, Osaka, Japan). The primers used were the forward primer 5'-CCATCTCATCCCTGCGTGTCTCCGACTCAGTGCTAGTCAGCCCTAYGGGGY GCASCAG-3' and reverse primer 5'-CCTATCCCCTGTGTGCCTTGGCAGTCTCAGGGCCATGCACYWCYTCTC-3'. The underlined sequences target a region of the archaeal 16S rRNA gene (Gantner et al., 2011). The sequences in italics are bar code sequences. The rest of the primer sequences were adaptor sequences. The partial sequence of the 16S rRNA gene was amplified in the reaction mixture by 30 cycles of $98{ }^{\circ} \mathrm{C}$ for $10 \mathrm{sec}, 55^{\circ} \mathrm{C}$ for $30 \mathrm{sec}$, and $68{ }^{\circ} \mathrm{C}$ for 22 sec, following initial denaturation at $94{ }^{\circ} \mathrm{C}$ for $2 \mathrm{~min}$. The amplicons were sequenced using the Roche GS FLX+ system by Hokkaido System Science (Sapporo, Hokkaido, Japan).

\section{Results and discussion}

3.1. Selection of marine sediments suitable for methane fermentation of seaweed under saline conditions 
Various marine sediments were cultured anaerobically with the brown alga under seawater salinity. In the batch culture, all of the sediments produced VFAs as intermediate metabolites and then produced methane (Table 2). Among the sediments, sediments 1 and 5 almost completely consumed the VFAs to produce methane at $27 \mathrm{~d}$; the other sediments partially converted the VFAs to methane during cultivation. Among the sediments, sediment 1 had already produced methane at $18 \mathrm{~d}$. In contrast, methane production by mesophilic granules collected from an anaerobic digester for wastewater discharged from a food-manufacturing factory was significantly inhibited by salt with accumulation of VFAs. In contrast, the granules completely converted VFAs other than propionate to methane without salt, indicating that the granules were not resistant to salt. These experiments suggested that a complete set of the microbial community for methane production at seawater salinity was contained in marine sediments, especially in the sediments 1 and 5, but not in mesophilic granules; thus, these sediments can be used for methane fermentation of marine macroalgae under saline conditions.

3.2. Methane production from brown alga by the selected marine sediment

Marine sediments are expected to be a promising microbial source for methane production from seaweed under saline conditions. Therefore, a detailed time profile was obtained for methane production from seaweed by using marine sediment 1 , which had the ability to produce methane the fastest (Fig. 1a). The mannitol contained in the brown alga was rapidly fermented to mainly acetate, butyrate, and ethanol. Propionate and hydrogen were also detected. After mannitol consumption, the amount of hydrogen, 
ethanol, and butyrate decreased, whereas the amount of acetate and propionate increased. The propionate concentration was highest at $4.5 \mathrm{~d}$ and then gradually decreased. In contrast, the acetate concentration continued to increase, possibly through degradation of the alginate contained in the brown alga. The acetate concentration peaked at $8.5 \mathrm{~d}$ and then began to decrease.

Methane was produced in the early phase of the culture by hydrogenotrophic methanogens because the hydrogen produced was consumed during this phase. After 8.5 d, methane was rapidly produced together with acetate and propionate consumption, suggesting activation of acetoclastic methanogens in the marine sediment. All substances detected in the experiments were completely consumed to produce methane at a concentration of $25.8 \mathrm{mM}$ at $27 \mathrm{~d}$. In this culture, the methane yield from seaweed was $0.42 \mathrm{~g}$-COD/g-COD. It should be noted that this yield was obtained under saline conditions but still comparable to that in a previous report of methane production from seaweed under reduced salinity (Hinks et al., 2013). This finding clearly demonstrates the effectiveness of the marine sediment as a methanogenic microbial community under saline conditions.

To determine whether the methane production from the brown alga by marine sediments was sustainable, the culture was diluted to $10 \%(\mathrm{v} / \mathrm{v})$ with fresh medium containing the alga and salt to conduct subculture (Fig. 1b). In the early phase of the subculture, mannitol, which was the major component of the alga, was rapidly consumed, and methane was produced as observed in the primary culture. However, in the subculture, methane production was temporary even after $8.5 \mathrm{~d}$ of cultivation with accumulation of acetate. Propionate also accumulated, and butyrate consumption was delayed relative to that in the primary culture. These results indicate that acetoclastic 
methanogenesis and acetogenesis from propionate and butyrate were not sustainable, whereas VFA production and hydrogenotrophic methanogenesis were sustainable. In general, the growth rates of acetogen and acetoclastic methanogens are slow (Xu et al., 2012). Thus, the lack of sustainability in acetogenesis and acetoclastic methanogenesis in the subculture probably resulted from the low population of those microbes. The population of the microbes in the subculture may not have reached that in the primary culture.

To determine why methane production was inhibited, the archaeal communities were analyzed after $27 \mathrm{~d}$ of cultivation of the primary and secondary cultures. A portion of the archaeal 16S rRNA gene was amplified, and the amplicons were sequenced by a next generation sequencer. In the primary culture, acetoclastic methanogens belonging to the Methanosarcina genus dominated. The proportion of sequence reads of Methanosarcina genus was $93.0 \%$ of 17407 reads. In contrast, hydrogenotrophic methanogens belonging to the Methanococcus genus were abundant in the subculture. The ratio of reads for Methanococcus genus was $89.5 \%$ of a total of 17895 reads, whereas Methanosarcina genus comprised only $1.6 \%$ of the total reads. These results suggest that in the subculture, the population of acetoclastic methanogens remained small, resulting in a failure to completely convert VFAs, especially acetate.

3.3. Microbial activity of marine sediments at each step of methane production

Methane fermentation is a complex process involving at least three types of microorganisms performing hydrolysis/acidogenesis, $\left(\mathrm{H}_{2}\right.$-forming) acetogenesis, and methanogenesis (McCarty \& Smith, 1986). To determine the methanogenic potential of 
marine sediments under saline conditions, the substrate conversion rates were measured at each step of methane production by the marine sediments and mesophilic granules (Table 3).

In the mesophilic granules under non-saline conditions, activity at all steps was detected as expected, although the rate of propionate oxidation was low. In contrast, in the presence of $3 \% \mathrm{NaCl}$, although hydrogenotrophic methane production was highly tolerant to high salinity, hydrolysis/acidogenesis and oxidation of butyrate in acetogenesis were significantly inhibited, and oxidation of propionate and acetoclastic methanogenesis was completely inhibited. This result clearly demonstrates that mesophilic granules developed in non-saline conditions are not feasible for efficient methane fermentation under saline conditions.

In contrast, the marine sediments tested had high activity throughout the steps of methane production under saline conditions, except in hydrolysis/acidogenesis and hydrogenotrophic methanogenesis, relative to the mesophilic granules. Of the sediments, sediments 1 and 5 showed activity in all steps of methane fermentation of the seaweed under saline conditions, indicating that the sediments contained a complete set of microbes, whereas the other three sediments did not show acetogenesis from propionate. This result agrees with the results of the preliminary experiments in Table 1 and the time profile in Fig. 1a. The present results strongly demonstrate that marine sediments can be used as microbial sources for effective methane production from salt-containing organic matter such as brown algae without dilution.

It was of concern that all marine sediments showed low activity in acetoclastic methanogenesis and propionate oxidation. Indeed, the subculture significantly weakened acetoclastic methanogenesis and propionate oxidation (Fig 1b), suggesting 
low microorganism growth rates compared to the other steps. This speculation is supported by the next generation sequence analysis of the archaeal community as mentioned above. The major VFA produced from the brown alga was acetate as shown in Fig. 1a, suggesting that acetoclastic methanogenesis was the rate-limiting step in methane production from brown algae. To overcome this constraint and practically use the marine microbial community, the microbial physiology of a variety of marine methanogenic microbes needs to be elucidated to optimize them further, and the appropriate bioreactor configuration for high-throughput treatment needs to be determined. Such investigations are now in progress.

\section{Conclusions}

In this study, various marine sediments were evaluated as microbial sources for methane production from brown alga under saline conditions. One of the tested marine sediments completely converted the produced volatile fatty acids to methane in a short period although acetoclastic methanogenesis seemed to be the limiting step. Compared to the activity of a conventional methanogenic microbial community, the marine sediments had high activity in all of the methane production steps except hydrolysis/acidogenesis and hydrogenotrophic methanogenesis. The result demonstrates the feasibility of using a marine microbial community for efficient methane fermentation of salt-containing brown algae without dilution.

\section{Acknowledgments}


This work was supported by the Core Research of Evolutional Science and Technology program (CREST) from the Japan Science and Technology Agency (JST).

\section{References}

1. Abouelenien, F., Fujiwara, W., Namba, Y., Kosseva, M., Nishio, N., Nakashimada, Y., 2010. Improved methane fermentation of chicken manure via ammonia removal by biogas recycle. Bioresour. Technol. 101, 6368-6373.

2. Demirbas, A., 2010. Use of algae as biofuel sources. Energy Conv. Manage. 51, $2738-2749$.

3. Feijoo, G., Soto, M., Méndez, R., Lema, J.M., 1995. Sodium inhibition in the anaerobic digestion process: antagonism and adaptation phenomena. Enzyme Microb. Technol. 17, 180-188.

4. Gantner, S., Andersson, A.F., Alonso-Sáez, L., Bertilsson, S., 2011. Novel primers for 16S rRNA-based archaeal community analyses in environmental samples. J. Microbiol. Methods 84, 12-18.

5. Hinks, J., Edwards, S., Sallis, P.J., Caldwell, G.S., 2013. The steady state anaerobic digestion of Laminaria hyperborea - Effect of hydraulic residence on biogas production and bacterial community composition. Bioresour. Technol. 143, 221-230.

6. Jung, K.A., Lim, S.R., Kim, Y., Park, J.M., 2013. Potentials of macroalgae as feedstocks for biorefinery. Bioresour. Technol. 135, 182-190.

7. McCarty, P.L., Smith, D.P., 1986. Anaerobic wastewater treatment. Environ. Sci. Technol. 20, 1200-1206. 
8. Roesijadi, G., Jones, S.B., Snowden-Swan, L.J., Zhu, Y., 2010. Macroalgae as a biomass feedstock: a preliminary analysis. Pacific Northwest National Laboratory. 9. Schramm, W., Lehnberg, W., 1984. Mass culture of brackish-water-adapted seaweeds in sewage-enriched seawater. II: Fermentation for biogas production. Hydrobiologia 116, 282-287.

10. Shi, X., Lefebvre, O., Ng, K.K., Ng, H.Y., 2014. Sequential anaerobic-aerobic treatment of pharmaceutical wastewater with high salinity. Bioresour. Technol. 153, 79-86.

11. Wei, N., Quarterman, J., Jin, Y.-S., 2013. Marine macroalgae: an untapped resource for producing fuels and chemicals. Trends Biotechnol. 31, 70-77.

12. Xu, S.Y., Karthikeyan, O.P., Selvam, A., Wong, J.W.C., 2012. Effect of inoculum to substrate ratio on the hydrolysis and acidification of food waste in leach bed reactor. Bioresour. Technol. 126, 425-430. 


\section{Figure captions}

Fig. 1. Methane production from brown alga using marine sediment 1 before (a) and after (b) subculture. Closed diamonds, mannitol; closed triangles, formate; closed squares, acetate; closed circles, propionate; open diamonds, butyrate; open triangles, ethanol; open squares, hydrogen; open circles, methane. Data points and error bars represent mean values and standard deviations of triplicates, respectively. 


\section{Table 1}

Microbial sources used in this study.

\begin{tabular}{|c|c|c|c|}
\hline Microbial source & Origin & $\mathrm{TS}^{\mathrm{a}}$ content $(\%)$ & $\mathrm{VS}^{\mathrm{b}}$ content $(\%)$ \\
\hline Mesophilic granule & $\mathrm{UASB}^{\mathrm{d}}$ reactor & 5.5 & 4.3 \\
\hline$M S^{c}-1$ & Ariake sea & 34.7 & 3.6 \\
\hline MS-2 & Tokyo Bay site A & 79.3 & 2.0 \\
\hline MS-3 & Tokyo Bay site B & 69.0 & 1.4 \\
\hline MS-4 & Tokyo Bay site C & 67.3 & 2.9 \\
\hline MS-5 & Hiroshima Bay & 55.9 & 5.0 \\
\hline
\end{tabular}

${ }^{\mathrm{a}}$ Total solid; ${ }^{\mathrm{b}}$ volatile solid; ${ }^{\mathrm{c}}$ marine sediment; ${ }^{\mathrm{d}}$ upflow anaerobic sludge blanket. 


\section{Table 2}

Production of volatile fatty acids and methane from seaweed by marine sediments.

\begin{tabular}{|c|c|c|c|c|c|c|c|c|c|}
\hline \multirow{2}{*}{$\begin{array}{l}\text { Microbial } \\
\text { source }\end{array}$} & \multirow[t]{2}{*}{$\mathrm{NaCl}$} & \multicolumn{2}{|c|}{$\begin{array}{l}\text { Acetate } \\
(\mathrm{mM})\end{array}$} & \multicolumn{2}{|c|}{$\begin{array}{l}\text { Propionate } \\
\qquad(\mathrm{mM})\end{array}$} & \multicolumn{2}{|c|}{$\begin{array}{c}\text { Butyrate } \\
(\mathrm{mM})\end{array}$} & \multicolumn{2}{|c|}{$\begin{array}{c}\text { Methane } \\
\text { (\% in headspace) }\end{array}$} \\
\hline & & $18 \mathrm{~d}$ & $27 \mathrm{~d}$ & $18 \mathrm{~d}$ & $27 d$ & $18 \mathrm{~d}$ & $27 d$ & $18 \mathrm{~d}$ & $27 d$ \\
\hline \multirow{2}{*}{$\begin{array}{l}\text { Mesophilic } \\
\text { granule }\end{array}$} & - & 18.4 & $\mathrm{ND}^{\mathrm{b}}$ & 1.4 & 2.1 & ND & ND & 16.8 & 32.2 \\
\hline & + & 9.2 & 17.3 & 2.9 & 3.0 & 4.9 & 0.8 & 6.6 & 8.7 \\
\hline $\mathrm{MS}^{\mathrm{a}}-1$ & + & 0.5 & ND & ND & ND & ND & ND & 23.7 & 24.9 \\
\hline MS-2 & + & 19.8 & 19.1 & 7.7 & 7.8 & ND & ND & 5.6 & 6.4 \\
\hline MS-3 & + & 14.9 & 15.6 & 5.3 & 5.3 & ND & ND & 4.4 & 4.5 \\
\hline MS-4 & + & 20.1 & 13.2 & 5.8 & 6.0 & 0.7 & ND & 9.2 & 15.7 \\
\hline MS-5 & + & 13.4 & 0.7 & 1.6 & ND & ND & ND & 16.2 & 25.7 \\
\hline
\end{tabular}

${ }^{\mathrm{a}}$ Marine sediment; ${ }^{\mathrm{b}}$ not detected. 


\section{Table 3}

Activity of marine sediments at each step of methane production at seawater salinity.

\begin{tabular}{|c|c|c|c|c|c|c|}
\hline \multirow{3}{*}{$\begin{array}{l}\text { Microbial } \\
\text { source }\end{array}$} & \multirow{3}{*}{$\mathrm{NaCl}$} & \multicolumn{5}{|c|}{ Conversion activity (mg-COD/g-VS/d) } \\
\hline & & \multirow{2}{*}{$\begin{array}{l}\text { Hydrolysis/ } \\
\text { acidogenesis }\end{array}$} & \multicolumn{2}{|c|}{ Acetogenesis } & \multicolumn{2}{|c|}{ Methanogenesis } \\
\hline & & & Propionate & Butyrate & $\mathrm{H}_{2}-\mathrm{CO}_{2}$ & Acetate \\
\hline \multirow{2}{*}{$\begin{array}{l}\text { Mesophilic } \\
\text { granule }\end{array}$} & - & 1,735 & 17 & 184 & 3,250 & 54 \\
\hline & + & $608(35.0)^{\mathrm{a}}$ & $\mathrm{ND}^{\mathrm{b}}(0)$ & $27(14.7)$ & $2,940(90.5)$ & $\mathrm{ND}(0)$ \\
\hline $\mathrm{MS}^{\mathrm{c}}-1$ & + & 405 & 79 & 121 & 1,170 & 48 \\
\hline MS-2 & + & 613 & ND & 554 & 3,480 & 102 \\
\hline MS-3 & + & 1,304 & ND & 1,476 & 5,690 & 79 \\
\hline MS-4 & + & 617 & ND & 719 & 1,130 & 45 \\
\hline MS-5 & + & 697 & 35 & 315 & 1,040 & 30 \\
\hline
\end{tabular}

${ }^{a}$ Percentage of remaining activity in the presence of $3 \% \mathrm{NaCl}$ compared to that without $\mathrm{NaCl}$ addition; ${ }^{\mathrm{b}}$ not detected during $60 \mathrm{~d}$ cultivation; ${ }^{\mathrm{c}}$ marine sediment. 

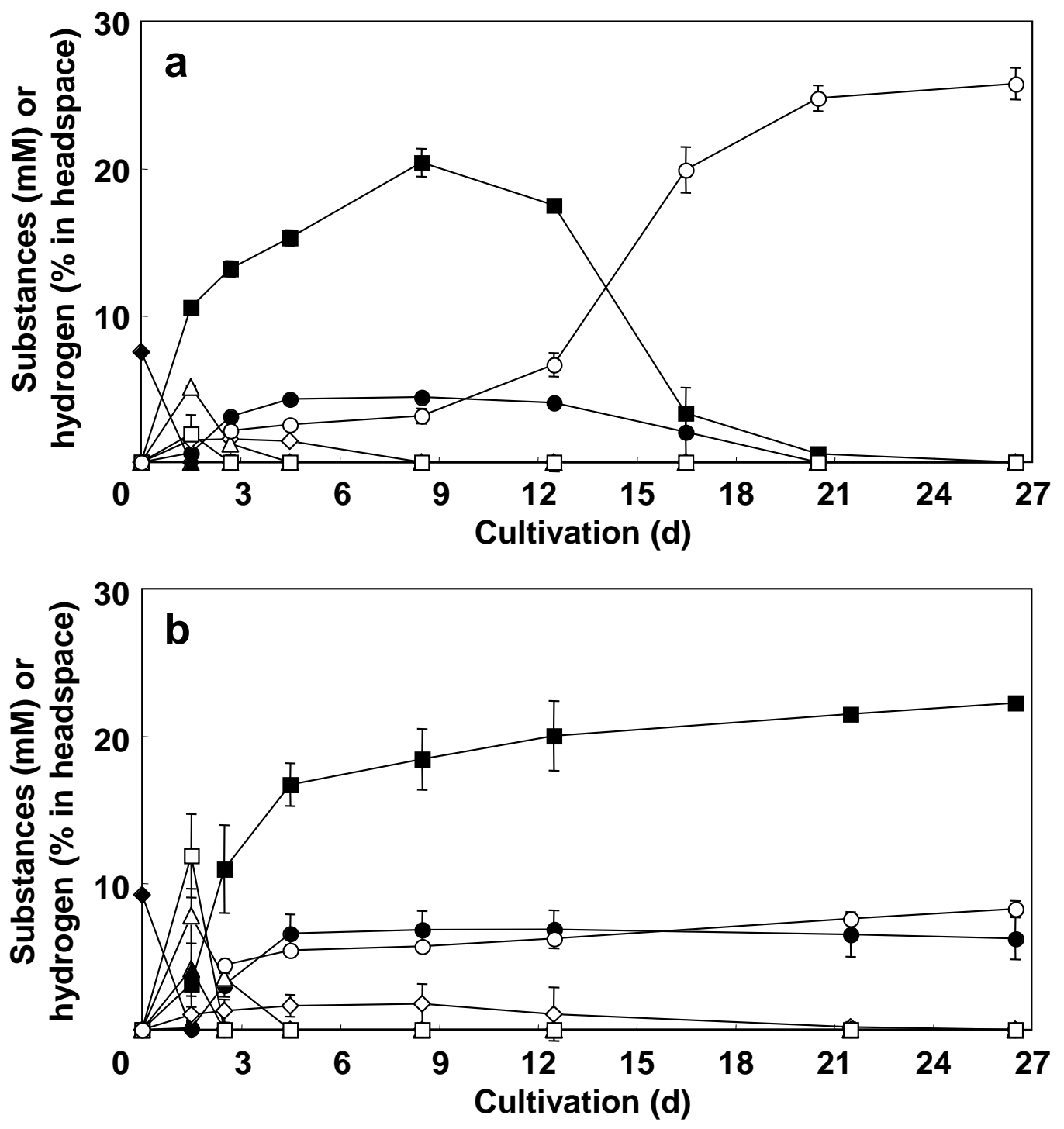

Fig. 1 